\title{
Association between Aminotransferase/alanine Aminotransferase Ratio and Cardiovascular Disease mortality in Patients on Peritoneal Dialysis
}

\section{Xiaoran Feng}

Nanchang University

\section{Yueqiang Wen}

Guangzhou Medical University

\section{FenFen Peng}

Southern Medical University

Niansong Wang

Shanghai Jiao Tong University

\section{Xiaojiang Zhan}

Nanchang University

Xianfeng Wu ( $\nabla$ xianfengwu2@163.com )

Shanghai Jiao Tong University

\section{Research article}

Keywords: Aspartate aminotransferase/alanine aminotransferase ratio, Cardiovascular disease, Mortality, Peritoneal dialysis

Posted Date: January 22nd, 2020

DOI: https://doi.org/10.21203/rs.2.21568/v1

License: (9) This work is licensed under a Creative Commons Attribution 4.0 International License.

Read Full License

Version of Record: A version of this preprint was published at BMC Nephrology on June 1st, 2020. See the published version at https://doi.org/10.1186/s12882-020-01840-7. 


\section{Abstract}

Objectives Elevated aspartate aminotransferase/alanine aminotransferase (AST/ALT) ratio is an independent risk factor for cardiovascular disease (CVD) among the general population. However, an association between AST/ALT ratio and CVD mortality in patients on peritoneal dialysis (PD) has received little attention.

Methods A total of 2224 incident PD patients from multi-centers were enrolled from November 1, 2005, to June 30, 2017, in this retrospective cohort study. The primary endpoint was CVD mortality. Eligible patients were divided into high and normal groups according to the AST/ALT ratio cut-off for CVD mortality with the receiver operating characteristic (ROC) curve. The associations between the AST/ALT ratio and CVD mortality were evaluated by the Cox regression model.

Results Of eligible 1579 patients with a mean age of $49.3 \pm 14.6$ years, $55.4 \%$ of patients were male, $18.1 \%$ of patients had diabetes, and $64.2 \%$ of patients had hypertension. The prevalence of a high AST/ALT ratio was $76.6 \%$ in the cohort population. During a follow-up period with 4659.6 patient-years, 316 patients died, of which 193 (61.1\%) deaths were caused by CVD episodes. The incidence of CVD mortality in the high group was significantly higher than that in the normal group $(13.1 \%$ versus $9.2 \%, P=0.024)$. Cumulative CVD mortality rates were significantly different between the two groups by Kaplan-Meier analysis [hazards ratio $(\mathrm{HR})=1.50,95 \%$ confidence index $(\mathrm{Cl}) 1.09-2.07, \mathrm{P}=0.014$ ]. After adjusting for confounding factors, a higher AST/ALT ratio was independently associated with an increased risk of CVD mortality compared with their counterparts $(\mathrm{HR}=1.43,95 \% \mathrm{Cl} 1.08-2.41, \mathrm{P}=0.002)$.

Conclusions PD patients with high baseline AST/ALT ratio levels may be at a significant risk of CVD mortality.

\section{Introduction}

Cardiovascular disease (CVD) represents the leading cause of death in peritoneal dialysis (PD) patients, accounting for up to $40 \%-60 \%$ of deaths $[1,2]$. Traditional risk factors, such as diabetes, hypertension, dyslipidemia, and a history of CVD, account for up to $50 \%$ of CVD in dialysis patients. At the same time, renal specific markers, including anemia, disordered bone mineral metabolism, and oxidative stress, also likely contribute to the total CVD burden in these patients [3-7]. Therefore, exploring new non-traditional risk factors for CVD episodes may be beneficial to further improve the prognosis of PD patients.

Aminotransferase is a well-known marker for liver injury and is composed of alanine aminotransferase (ALT) and aspartate aminotransferase (AST). ALT is only located in the liver, but AST is in both the liver and myocardial tissue [8]. A more significant increase in AST characterizes the elevation of aminotransferase in CVD compared with ALT, which presented an increased AST/ALT ratio in patients with CVD $[9,10]$. A previous study showed that a history of CVD rate was higher in subjects with a high AST/ALT ratio than in those with a normal AST/ALT ratio, which suggested that an elevated AST/ALT ratio may reflect cardiac load and damage and the presence of latent CVD [11, 12]. A previous study with 
a 10-year follow-up reported that the increased AST/ALT ratio, as a new non-traditional risk for CVD, was an independent predictor of CVD mortality in the general population who participated in a communitybased health check-up, [8]. Another study from the United Kingdom showed that elevated AST/ALT ratio is significantly associated with an increased risk of developing CVD in men with no history of CVD at baseline [13]. It was noteworthy that patients on dialysis had reduced serum levels of aminotransferases $[14,15]$, whereas whether the AST/ALT ratio was an independent predictor of CVD mortality in dialysis patients remains unknown. In the present study, the aim of this study was to evaluate the association between the AST/ALT ratio and CVD mortality in PD patients.

\section{Materials And Methods}

\section{Study Design and Population}

All 2224 incident PD patients who were followed up at four PD centers from November 1, 2005, to February 28, 2017, were enrolled at this multi-center retrospective cohort study. Inclusion criteria were age $\geq 18$ years at the start of PD and survival for $\geq$ three months from the first PD therapy. Previous studies reported that the increased AST/ALT ratio is due to induction by alcohol consumption and cardio-hepatic interaction [16, 17]. Thus, patients were excluded from the study if they had current drinking, had been diagnosed with a history of CVD, chronic liver disease, or AST or AST values more than two times higher normal values. The study is consistent with the ethical principles of the Declaration of Helsinki and was approved by the Human Ethics Committee of each research center. Written informed consent was obtained from all eligible patients.

Baseline demographic data included age, sex, Charlson comorbidity index (CCI), diabetes, hypertension, hyperlipidemia, gastrointestinal bleeding, current smoking, and medication use. Clinical and biochemical data at the initiation of PD included body mass index (BMI), ejection fraction, estimated glomerular filtration rate (eGFR), hemoglobin, serum albumin, AST, ALT, total bilirubin, cholesterol, triglycerides, highdensity lipoprotein (HDL), low-density lipoprotein (LDL), high-sensitivity C-reactive protein (hs-CRP), Nterminal -prohormone BNP (NT-proBNP), and 24-hr urine output. All baseline data were obtained during the first month of PD.

The primary and secondary endpoints were CVD and all-cause mortality, respectively. The PD team consisted of two nephrologists at each center who reviewed the details of individual medical records and identified the causes of death. If death had two or more potential causes, we generally ascribed the death to the primary cause for hospitalization or the initial presenting condition. If a patient died within three months of transfer to hemodialysis therapy, he or she was not censored because the early mortality was considered to reflect health status during the period of failing PD treatment. All patients were followed up until cessation of PD, death, or May 31, 2017. The censored data included switching to hemodialysis, renal transplantation, moving to another center, loss to follow-up, or still at our PD centers with a followup duration of 5 years. All patients received continuous ambulatory PD treatment. Conventional PD 
solutions (Dianeal 1.5\%, 2.5\%, or 4.25\% dextrose; Baxter Healthcare, Guangzhou, China), Y sets, and twin bag systems were used in all PD patients.

\section{Definitions}

CVD was defined as coronary events, arrhythmias, sudden cardiac death, congestive heart failure, or cerebrovascular events [18]. Chronic liver diseases are defined as alcoholic and non-alcoholic liver disease, autoimmune liver disease, hepatitis $B$ or $C[13,19,20]$. We defined aminotransferase elevation as any value above normal of ALT or AST based on a recent, nationally representative the United States survey (AST >40 IU/L, or ALT >43 IU/L) [21]. The comorbidity score was determined according to the $\mathrm{CCl}$, which is one of the most commonly used comorbidity models [22]. Baseline residual renal function was assessed by eGFR using the Chronic Kidney Disease Epidemiology Collaboration creatinine equation [23].

\section{Statistical analysis}

Data were expressed as mean \pm standard deviation, percentages, or median $\left(25^{\text {th }}-75^{\text {th }}\right.$ percentile). All eligible patients were divided into high and normal groups according to the AST/ALT ratio cut-off for CVD mortality with the receiver operating characteristic (ROC) curve. Comparisons of baseline parameters between two groups were conducted with $t$-tests for continuous normally distributed variables, MannWhitney test for continuous non-normally distributed data and $\chi^{2}$ analyses for categorical data. Logistic regression analyses were conducted to evaluate the association between baseline variables and high AST/ALT ratio. Variables with $\mathrm{P}<0.05$ in the univariate Logistic regression analysis were picked into a multivariate-adjusted Logistic regression model. Survival was estimated using the Kaplan-Meier curve, and differences were examined using the log-rank test. The associations between the AST/ALT ratio and CVD and all-cause mortality were evaluated by Cox proportional hazards regression. Unadjusted association was first examined, followed by adjustments for age, sex and $\mathrm{CCl}$, current smoking, and medication use, including angiotensin-converting enzyme inhibitor/angiotensin receptor blocker (ACEI/ARB), calcium antagonist, $\beta$-blocker, diuretic and statin use. Next, BMI, eGFR, hemoglobin, albumin, total bilirubin, cholesterol, triglycerides, hs-CRP, and 24-hr urine output were added to examine whether the association of the AST/ALT ratio with endpoints was independent of confounding factors. The results of the Cox regression models were presented as the hazard ratio (HR) and the $95 \%$ confidence interval $(\mathrm{Cl})$. A value of $\mathrm{P}<0.05$ was considered statistically significant. Statistical analyses were performed using GraphPad software 8.0 (GraphPad Prism Software Inc., San Diego, California) and the R package 3.6.0 (https://www.r-project.org/).

\section{Results}

\section{Baseline characteristics}

A total of 2224 incident PD patients were enrolled in the present study, of whom ten patients younger than 18 years, 84 patients on PD $<3$ months, 35 with current drinking, 279 with a history of CVD, 134 with chronic liver disease, 77 without baseline AST/ALT ratio, and 26 with AST or ALT values $\geq$ two times 
higher than normal values were excluded. The remaining 1579 patients with baseline AST/ALT ratio were eligible for the present analysis (Figure 1). Of 1579 patients with $\mathrm{CCl}$ of $3.87 \pm 1.66$, the mean age was $49.3 \pm 14.6$ years, $55.4 \%$ were male sex, $18.1 \%$ had diabetes, $64.2 \%$ had hypertension, and $16.2 \%$ had hyperlipidemia.

In ROC curve analysis, the AST/ALT ratio (area under curve $=0.75,95 \% \mathrm{Cl} 0.72-0.77 ; \mathrm{p}<0.001$ ) was found to be a significant predictor of CVD mortality with a sensitivity $(79.4 \%)$ and specificity $(75.8 \%)$. The cutoff of the AST/ALT ratio for CVD mortality was 1.0 in the cohort population. A total of $1210(76.6 \%)$ patients were in the high group and 369 (23.4\%) patients in the normal group. The baseline characteristics of the study population are shown in Table 1. Patients with high AST/ALT ratio were older $(P<0.001)$, likely to be female sex $(P<0.001)$, had higher frequency of hyperlipidemia $(P=0.029)$ and statin use $(P=0.037)$, had higher $C C I(P<0.001)$ and LDL $(P=0.038)$, and had a lower ALT values $(P<0.001)$ as compared to their counterparts.

\section{The high AST/ALT ratio}

The prevalence of the high AST/ALT ratio was 76.6\% (74.5\%-78.7\%) in the cohort population (Figure 2). Univariate Logistic analysis found that age $(P<0.001)$, female sex $(P<0.001), C C l(P<0.001)$, hyperlipidemia $(P=0.026)$, statin use $(P=0.035)$ and LDL $(P=0.039)$ were associated with high AST/ALT ratio (Table 2). Multivariate Logistic analysis showed that older age (increased pre one year, HR=1.02, $95 \% \mathrm{Cl} 1.01-1.03, \mathrm{P}<0.001)$ and female $(\mathrm{HR}=3.04,95 \% \mathrm{Cl} 2.31-4.06, \mathrm{P}<0.001)$ were independently associated with the high AST/ALT ratio.

\section{Baseline AST/ALT ratio and endpoints}

The median follow-up period was 4659.6 patient-years. By the end of this study, $316(20.0 \%)$ patients had died, $106(6.7 \%)$ patients had undergone renal transplantation, 247 (15.6\%) patients had transferred to hemodialysis, 18 (1.1\%) patients had transferred to other PD centers, and $60(3.8 \%)$ patients had been lost to follow-up; the remaining 832 (52.7\%) patients were still followed at these PD centers. Of 316 deaths, 193 (61.1\%) deaths were caused by CVD episodes. The CVD mortality incidence was $13.1 \%$ $(95 \% \mathrm{Cl} 11.2 \%-15.0 \%)$ and $9.2 \%(95 \% \mathrm{Cl} 6.2 \%-12.2 \%)$ in the high and normal groups, respectively $(\mathrm{P}=0.024$, Figure $3 \mathrm{~A})$, and the all-cause mortality rates was $21.4 \%(95 \% \mathrm{Cl} 19.1 \%-23.7 \%)$ and $15.4 \%(95 \% \mathrm{Cl}$ $11.7 \%-19.2 \%$ ) in the high and normal groups, respectively ( $P=0.001$, Figure $3 \mathrm{~B}$ ). The Kaplan-Meier estimates showed that the cumulative CVD and all-cause mortality incidence were significantly different between two AST/ALT ratio groups $(\mathrm{HR}=1.50,95 \% \mathrm{Cl} 1.09-2.07$, and $\mathrm{HR}=1.53,95 \% \mathrm{Cl} 1.16-1.93$, Figure $4 \mathrm{~A}$ and Figure $5 \mathrm{~A}$ ). At the end of 1,3 , and 5 years in this study, the incidence of CVD mortality was $8.1 \%$, $15.8 \%$, and $24.5 \%$ in the high group, and $6.1 \%, 10.2 \%$, and $15.2 \%$ in the normal group, respectively. The incidence of all-cause mortality was $12.7 \%, 26.7 \%$, and $32.6 \%$ in the normal group, and $9.8 \%, 17.8 \%$, and $20.7 \%$ in the high group, respectively.

The association between the baseline AST/ALT ratio and CVD and all-cause mortality is shown in Table 3. Crude Cox model analysis showed that a high AST/ALT ratio was associated with an increased risk of 
CVD and all-cause mortality $(\mathrm{HR}=1.63,95 \% \mathrm{Cl} 1.13-2.27 ; \mathrm{HR}=1.58,95 \% \mathrm{Cl} 1.18-2.10$, Model 1). Multivariate Cox model analysis found that patients with a high AST/ALT ratio carried a higher risk of CVD and allcause mortality ( $\mathrm{HR}=1.43,95 \% \mathrm{Cl} 1.08-2.41$, and $\mathrm{HR}=1.45,95 \% \mathrm{Cl} 1.13-2.37$, Model 3), even after adjusting for confounding factors.

\section{Subgroup analyses}

The prevalence of high AST/ALT ratio ranged from $68.4 \%(95 \% \mathrm{Cl} 65.3 \%-71.5 \%)$ to $86.9 \%(95 \% \mathrm{Cl}$ 84.3\%-89.3\%) among all subgroups (Figure 2). The prevalence of high AST/ALT ratio was significant difference between females and males $(P<0.001)$, those aged $\geq 65$ years and $<65$ years $(P<0.001)$, and hyperlipidemia and non-hyperlipidemia $(P=0.029)$. The incidence of CVD and all-cause mortality among subgroups were shown in Figure $3 \mathrm{~A}$, and $\mathrm{B}$. Male and non-hyperlipidemia with high a AST/ALT ratio had a significantly higher CVD mortality than their counterparts $(P=0.031$ and $P=0.013)$. Non-diabetes, hypertension, and non-hyperlipidemia with a high AST/ALT ratio had a significantly higher all-cause mortality than their counterparts $(P=0.010, P=0.001$, and $P=0.001)$. Survival analysis showed that the cumulative CVD mortality incidence between high and normal groups was a significant difference in the male and non-hyperlipidemia subgroups (Figure $4 \mathrm{~B}$ and C). The cumulative all-cause mortality incidence between high and normal groups was a significant difference in the non-diabetes, hypertension, and nonhyperlipidemia subgroups (Figure 5 B, C, and D). Adjusted HRs for CVD mortality were conducted in the male and non-hyperlipidemia subgroups, and for all-cause mortality in the non-diabetes, hypertension, and non-hyperlipidemia subgroups by the Cox regression models (Figure 6).

\section{Discussion}

In the present study, we found that higher baseline AST/ALT ratio may carry an increased risk of CVD and all-cause mortality in PD patients. Also, even though we excluded those patients with chronic liver disease, or a history of CVD, PD patients at the commencement of PD may have a higher prevalence of high AST/ALT ratio.

Aminotransferase, including AST and ALT, is a well-known marker for liver injury. AST is in both the liver and myocardial tissue, but ALT is only in the liver [8]. The elevation of the AST/ALT ratio is due to induction by alcohol consumption and cardio-hepatic interaction [16, 24]. A history of CVD prevalence was higher in subjects with a high AST/ALT ratio than in those with a low AST/ALT ratio [8]. These findings suggested that an elevated AST/ALT ratio may reflect cardiac load and damage, and the presence of underlying CVD. A longitudinal cohort study from Japan reported that the high AST/ALT ratio was an independent predictor of CVD and all-cause mortality in 3,494 Japanese subjects $>40$ years with a 10-year follow-up [8]. In this study, subjects were excluded due to end-stage real disease, incomplete data, or study withdrawal, but those with alcohol consumption or a history of CVD failed to be excluded. Therefore, these findings of this study may be less convincing due to selective bias. Another study from Italy reported that the AST/ALT ratio was independently associated with an increased risk of both CVD and all-cause mortality in 2529 type 2 diabetes patients with a 6-year follow-up. Patients with a known 
history of drug-induced liver injury, viral hepatitis, cirrhosis of any etiology, and hemochromatosis were also excluded, but those with a history of CVD failed to be excluded in this study [25]. More recently, a prospective cohort from the United Kingdom reported that an elevated AST/ALT ratio is significantly associated with an increased risk of developing CVD in men but not women [13]. A total of 29,316 subjects aged 25-84 years with no history of CVD at baseline were enrolled and followed up for ten years in this study. However, the AST/ALT ratio failed to confer any additional benefits in predictive accuracy for predicting CVD when included in standard primary care-based risk prediction tools such as Framingham Risk Scores. The major limitation was that patients with chronic liver disease were not excluded from this study. In the present study, to reduce selection bias, we excluded those current drinking, liver disease, and those with a history of CVD. We found that a higher AST/ALT ratio was independently associated with an increased risk for CVD and all-cause mortality. PD patients with a high AST/ALT ratio may have a 1.43-fold higher risk of CVD mortality and a 1.45-fold higher risk of all-cause mortality compared with their counterparts, even after adjustment for confounding factors. Subgroup analyses showed that a high AST/ALT ratio remained an independent predictor for CVD mortality in those male and non-hyperlipidemias, and all-cause mortality in those non-diabetes, hypertension, and nonhyperlipidemias. These findings suggested, along with previous studies, that PD patients with a higher AST/ALT ratio may have more CVD and all-cause involvement, and a preprocedural AST/ALT ratio, a widely available and inexpensive biomarker, might be helpful for risk stratification of CVD and all-cause mortality in PD patients.

A previous study reported that the prevalence of high AST/ALT ratio $\geq 1.0$ was $37.9 \%$ in 2529 type 2 diabetes patients with a 6-year follow-up [13]. Patients with chronic liver diseases were excluded, but those with a history of CVD were not excluded from this study, which may lead to an over-estimated prevalence of high AST/ALT ratio. To date, the prevalence of the high AST/ALT ratio in dialysis patients has received little attention. In the present study, we excluded those with chronic liver disease or a history of CVD, which may be considered as an essential reason to increase the AST/ALT ratio. Nonetheless, the prevalence of a high AST/ALT ratio was $76.6 \%$ in the cohort study and ranged from $68.4-86.9 \%$ among all subgroups. Thus, there might be a higher prevalence of high AST/ALT ratio in PD patients. These findings suggested, along with previous studies, that future studies should further investigate the prevalence of the AST/ALT ratio in dialysis patients and whether the prognosis of PD patients might be improved by the management of the high AST/ALT ratio.

ALT has potential value as a novel biomarker of aging [13]. Decreased ALT resulted from a reduced liver size and liver blood flow and was associated with aging, frailty, and higher mortality in the general elderly population [26, 27]. There is a correlation between ALT levels and the severity of renal failure [15].

Patients on dialysis had reduced serum levels of aminotransferases, which suggested that the ALT levels were reduced concomitantly with the progression of renal dysfunction $[14,15]$. In the present study, PD patients with a high AST/ALT ratio tended to have lower ALT levels. However, the association between ALT and clinical outcomes in PD patients received little attention. Future research should investigate the association between ALT and clinical outcomes in dialysis patients. 
There are several limitations to the present study. First, a retrospective study allows us to establish associations but not causal relationships. It was impossible for us to adjust all factors for CVD and allcause mortality, and the effect of residual confounding cannot be eliminated completely. Nonetheless, to reduce the effect of residual confounding on endpoints, we adjusted for significant risk factors for CVD and all-cause mortality. Second, PD patients usually took multiple drugs simultaneously due to other complications. So, it was difficult to determine which drugs may influence liver aminotransferase because of the interaction of drugs. Although we failed to exclude those patients whose liver aminotransferase may influence by multiple drugs, those with AST or ALT values $\geq$ two times higher than normal values were excluded. Thus, the effect of drugs on liver aminotransferase may be minimized. Third, rare chronic diseases such as hemochromatosis, which may influence aminotransferase activity, failed to be excluded in the present study. Fourth, we only evaluated baseline variables rather than changes over time in these variables of CVD and all-cause mortality. Finally, because PD patients were all Chinese in the present study, the results may not apply to other ethnic PD patients.

\section{Conclusions}

In conclusion, a high AST/ALT ratio at the initiation of PD was independently associated with an increased risk for CVD and all-cause mortality in PD patients. In addition, there may be a higher prevalence of high baseline AST/ALT ratio in PD patients. Fortunately, since laboratory assays for the AST/ALT ratio are common, readily available, and inexpensive, the AST/ALT ratio could be a promising parameter to identify PD patients at high risk for CVD and all-cause mortality. Future research should further investigate the prevalence of the AST/ALT ratio in PD patients and prospectively evaluate whether the prognosis of PD patients may be improved by the management of the AST/ALT ratio.

\section{List Of Abbreviations}

AST/ALT, aspartate aminotransferase/alanine aminotransferase; CVD, cardiovascular disease; CCI, Charlson comorbidity index; ACEI/ARB, angiotensin-converting enzyme inhibitor/angiotensin receptor blocker; BMI, body mass index; eGFR, estimated glomerular filtration rate; HDL, high-density lipoprotein; LDL, low-density lipoprotein; high-sensitivity C-reactive protein (hs-CRP); NT-pro-BNP, N-terminal prohormone BNP.

\section{Declarations}

Ethics approval and consent to participate: The study was consistent with the ethical principles of the Declaration of Helsinki and was approved by the Human Ethics Committee of the Second Affiliated Hospital of Guangzhou Medical University, Zhujiang Hospital of Southern Medical University, Jiujiang No. 1 People's Hospital, Affiliated Sixth People's Hospital, Shanghai Jiao Tong University, and the First Affiliated Hospital of Nanchang University. Written informed consent was obtained from all participants. 
Consent for Publication: All authors have approved the submitted version. All authors have agreed both to be personally accountable for the author's own contributions and to ensure that questions related to the accuracy or integrity of any part of the work, even ones in which the author was not personally involved, are appropriately investigated, resolved, and the resolution documented in the literature.

Availability of data and material: Availability of data and materials: The datasets used and/or analyzed during the current study are available from the corresponding author on reasonable request.

Competing interests: The authors declare that they have no competing interests.

Funding: No.

Authors' contributions: Xiaoran Feng, contributions to the conception, interpretation of data, and drafted the work; FenFen Peng, the acquisition, analysis and interpretation of data; Yueqiang Wen, the acquisition, analysis and interpretation of data; Niansong Wang, contributions to the conception and design of the work; Xiaojiang Zhan, contributions to the conception and design of the work; Xianfeng Wu, contributions to the conception, design of the work, and revised it.

Acknowledgements: We express our gratitude to all patients who participated in the study.

\section{References}

1. Johnson DW, Dent H, Hawley CM, McDonald SP, Rosman JB, Brown FG, Bannister K, Wiggins KJ: Association of dialysis modality and cardiovascular mortality in incident dialysis patients. Clin $\mathrm{J} \mathrm{Am}$ Soc Nephrol 2009, 4(10):1620-1628.

2. Mehrotra R, Devuyst O, Davies SJ, Johnson DW: The Current State of Peritoneal Dialysis. J Am Soc Nephrol 2016, 27(11):3238-3252.

3. Kennedy R, Case C, Fathi R, Johnson D, Isbel N, Marwick TH: Does renal failure cause an atherosclerotic milieu in patients with end-stage renal disease? Am J Med 2001, 110(3):198-204.

4. Isbel NM, Haluska B, Johnson DW, Beller E, Hawley C, Marwick TH: Increased targeting of cardiovascular risk factors in patients with chronic kidney disease does not improve atheroma burden or cardiovascular function. Am Heart J 2006, 151(3):745-753.

\section{Zoccali C: Traditional and emerging cardiovascular and renal risk factors: an epidemiologic} perspective. Kidney Int 2006, 70(1):26-33.

6. Kaisar M, Isbel N, Johnson DW: Cardiovascular disease in patients with chronic kidney disease. A clinical review. Minerva Urol Nefrol 2007, 59(3):281-297.

7. Kaisar MO, Isbel NM, Johnson DW: Recent clinical trials of pharmacologic cardiovascular interventions in patients with chronic kidney disease. Rev Recent Clin Trials 2008, 3(2):79-88.

8. Yokoyama M, Watanabe T, Otaki Y, Takahashi H, Arimoto T, Shishido T, Miyamoto T, Konta T, Shibata $\mathrm{Y}$, Daimon $\mathrm{M}$ et al: Association of the Aspartate Aminotransferase to Alanine Aminotransferase Ratio 
with BNP Level and Cardiovascular Mortality in the General Population: The Yamagata Study 10-Year Follow-Up. Dis Markers 2016, 2016:4857917.

9. Fuhrmann V, Jager B, Zubkova A, Drolz A: Hypoxic hepatitis - epidemiology, pathophysiology and clinical management. Wien Klin Wochenschr2010, 122(5-6):129-139.

10. Henrion J: Hypoxic hepatitis. Liver Int 2012, 32(7):1039-1052.

11. Samsky MD, Patel CB, DeWald TA, Smith AD, Felker GM, Rogers JG, Hernandez AF: Cardiohepatic interactions in heart failure: an overview and clinical implications. J Am Coll Cardiol 2013, 61(24):2397-2405.

12. Lofthus DM, Stevens SR, Armstrong PW, Granger CB, Mahaffey KW: Pattern of liver enzyme elevations in acute ST-elevation myocardial infarction. Coron Artery Dis 2012, 23(1):22-30.

13. Weng SF, Kai J, Guha IN, Qureshi N: The value of aspartate aminotransferase and alanine aminotransferase in cardiovascular disease risk assessment. Open Heart 2015, 2(1):e000272.

14. Sette LH, Almeida Lopes EP: Liver enzymes serum levels in patients with chronic kidney disease on hemodialysis: a comprehensive review. Clinics (Sao Paulo) 2014, 69(4):271-278.

15. Liberato IR, Lopes EP, Cavalcante MA, Pinto TC, Moura IF, Loureiro Junior L: Liver enzymes in patients with chronic kidney disease undergoing peritoneal dialysis and hemodialysis. Clinics (Sao Paulo) 2012, 67(2):131-134.

16. Alonso A, Misialek JR, Amiin MA, Hoogeveen RC, Chen LY, Agarwal SK, Loehr LR, Soliman EZ, Selvin E: Circulating levels of liver enzymes and incidence of atrial fibrillation: the Atherosclerosis Risk in Communities cohort. Heart 2014, 100(19):1511-1516.

17. Torruellas C, French SW, Medici V: Diagnosis of alcoholic liver disease. World J Gastroenterol 2014, 20(33):11684-11699.

18. Wu J, Lei G, Wang X, Tang Y, Cheng H, Jian G, Wu X, Wang N: Asymptomatic hyperuricemia and coronary artery disease in elderly patients without comorbidities. Oncotarget 2017, 8(46):8068880699.

19. Nyblom H, Berggren U, Balldin J, Olsson R: High AST/ALT ratio may indicate advanced alcoholic liver disease rather than heavy drinking. Alcohol Alcohol 2004, 39(4):336-339.

20. Nyblom $\mathrm{H}$, Nordlinder $\mathrm{H}$, Olsson R: High aspartate to alanine aminotransferase ratio is an indicator of cirrhosis and poor outcome in patients with primary sclerosing cholangitis. Liver Int 2007, 27(5):694699.

21. loannou GN, Boyko EJ, Lee SP: The prevalence and predictors of elevated serum aminotransferase activity in the United States in 1999-2002. Am J Gastroenterol 2006, 101(1):76-82.

22. Charlson ME, Pompei P, Ales KL, MacKenzie CR: A new method of classifying prognostic comorbidity in longitudinal studies: development and validation. J Chronic Dis 1987, 40(5):373-383.

23. Zhang L, Wang F, Wang L, Wang W, Liu B, Liu J, Chen M, He Q, Liao Y, Yu X et al: Prevalence of chronic kidney disease in China: a cross-sectional survey. Lancet 2012, 379(9818):815-822. 
24. Kamimoto Y, Horiuchi S, Tanase S, Morino Y: Plasma clearance of intravenously injected aspartate aminotransferase isozymes: evidence for preferential uptake by sinusoidal liver cells. Hepatology 1985, 5(3):367-375.

25. Zoppini G, Cacciatori V, Negri C, Stoico V, Lippi G, Targher G, Bonora E: The aspartate aminotransferase-to-alanine aminotransferase ratio predicts all-cause and cardiovascular mortality in patients with type 2 diabetes. Medicine (Baltimore) 2016, 95(43):e4821.

26. Le Couteur DG, Blyth FM, Creasey HM, Handelsman DJ, Naganathan V, Sambrook PN, Seibel MJ, Waite LM, Cumming RG: The association of alanine transaminase with aging, frailty, and mortality. $J$ Gerontol A Biol Sci Med Sci 2010, 65(7):712-717.

27. Liu Z, Que S, Xu J, Peng T: Alanine aminotransferase-old biomarker and new concept: a review. Int J Med Sci 2014, 11(9):925-935.

\section{Tables}

Table 1. Baseline characteristics of patients stratified by baseline AST/ALT ratio. 


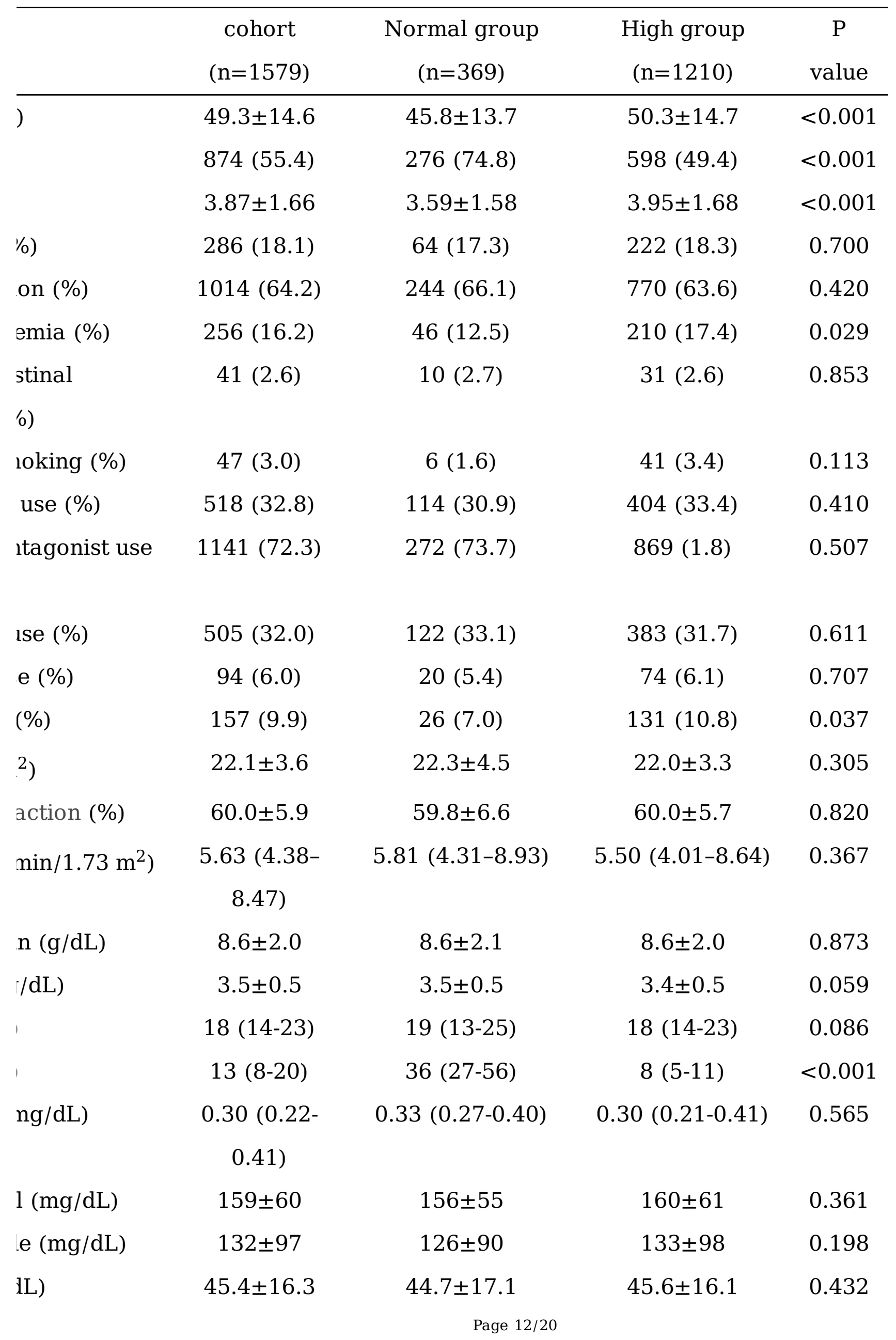




\begin{tabular}{lcccc} 
L) & $99.0 \pm 38.8$ & $95.1 \pm 37.0$ & $100.2 \pm 39.3$ & 0.038 \\
$\mathrm{~g} / \mathrm{L})$ & $4.03(1.97-$ & $2.25(0.85-19.5)$ & $4.25(2.04-14.20)$ & 0.209 \\
& $11.04)$ & & & \\
$\mathrm{P}(\mathrm{pg} / \mathrm{mL})$ & $2017(800-$ & $771(412-4835)$ & $2375(840-5760)$ & 0.824 \\
& $6545)$ & & & \\
& $851 \pm 534$ & $890 \pm 589$ & $839 \pm 527$ & 0.131 \\
\hline
\end{tabular}

AST/ALT, aspartate aminotransferase/alanine aminotransferase; CCI, Charlson comorbidity index; ACEI/ARB, angiotensin-converting enzyme inhibitor/angiotensin receptor blocker; BMI, body mass index; eGFR, estimated glomerular filtration rate; HDL, high-density lipoprotein; LDL, low-density lipoprotein; high-sensitivity C-reactive protein (hs-CRP); NT-pro-BNP, N-terminal -prohormone BNP.

Table 2. Predictors for high AST/ALT ratio by Logistic regression

\begin{tabular}{|c|c|c|c|c|}
\hline \multirow{2}{*}{ es } & \multicolumn{2}{|c|}{$\begin{array}{c}\text { Univariate Logistic } \\
\text { regression }\end{array}$} & \multicolumn{2}{|c|}{$\begin{array}{c}\text { Multivariate Logistic } \\
\text { regression }\end{array}$} \\
\hline & HR $(95 \% C I)$ & $P$ value & HR (95\%CI) & $P$ value \\
\hline creased pre 1 & $1.02(1.01-1.03)$ & $<0.001$ & $1.02(1.01-1.03)$ & $<0.001$ \\
\hline (yes/no) & $3.06(2.34-3.94)$ & $<0.001$ & $3.04(2.31-4.06)$ & $<0.001$ \\
\hline creased per 1 & $1.57(1.07-1.24)$ & $<0.001$ & - & - \\
\hline pidemia (yes/no) & $1.48(1.05-2.08)$ & 0.026 & - & - \\
\hline ise (yes/no) & $1.60(1.03-2.48)$ & 0.035 & - & - \\
\hline ıcreased per mg/l) & $1.02(1.01-1.03)$ & 0.039 & - & - \\
\hline
\end{tabular}

Variables with $\mathrm{P}<0.05$ in the univariate analysis were picked into the multivariate-adjusted model. 
AST/ALT, aspartate aminotransferase/alanine aminotransferase; CCI, Charlson comorbidity index; LDL, low-density lipoprotein.

Table 3. Adjusted hazards ratio for CVD and all-cause mortality using Cox regression models

\begin{tabular}{lccc}
\hline & Model 1 & Model 2 & Model 3 \\
\cline { 2 - 4 } & HR (95\%) & HR (95\%) & HR (95\%) \\
\hline rtality & $1.63(1.13-2.27)$ & $1.55(1.10-2.36)$ & $1.43(1.08-2.41)$ \\
mortality & $1.58(1.18-2.10)$ & $1.48(1.16-2.24)$ & $1.45(1.13-2.37)$ \\
\hline
\end{tabular}

Hazards ratio: high AST/ALT ratio vs. normal AST/ALT ratio. Model 1: unadjusted. Model 2: adjusted for age, sex, CCI, smoking, and medication use. Model 3: model 2 adjusted for BMI, eGFR, hemoglobin, albumin, bilirubin, cholesterol, triglycerides, hs-CRP, and 24-hr urine output.

AST/ALT, aspartate aminotransferase/alanine aminotransferase; CVD, cardiovascular disease; CCI, Charlson comorbidity index; BMI, body mass index; eGFR, estimated glomerular filtration rate; high-sensitivity C-reactive protein (hs-CRP).

\section{Figures}




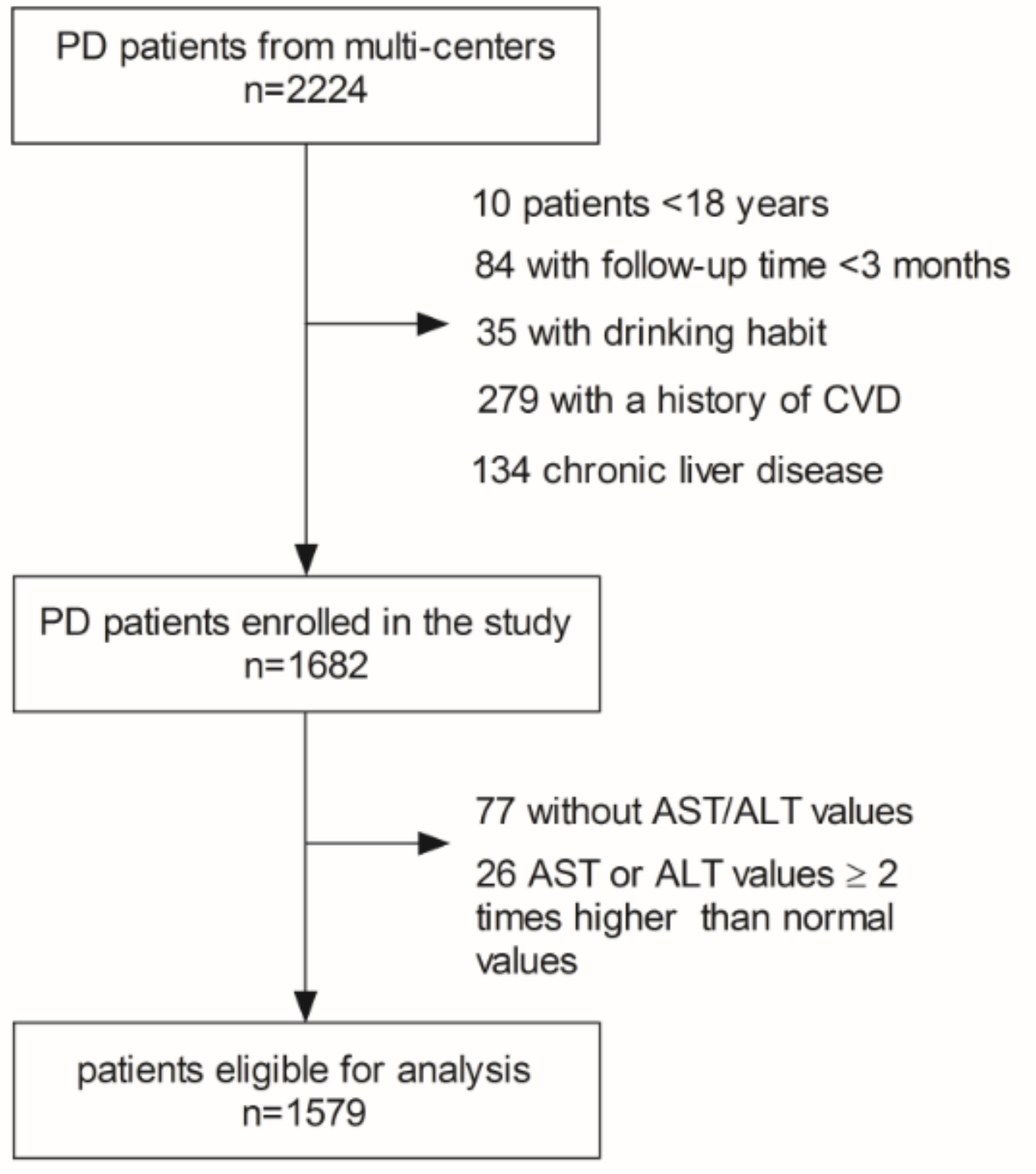

\section{Figure 1}

Patient flow in the study. PD, peritoneal dialysis; CVD, cardiovascular disease; AST/ALT, aspartate aminotransferase/alanine aminotransferase. 


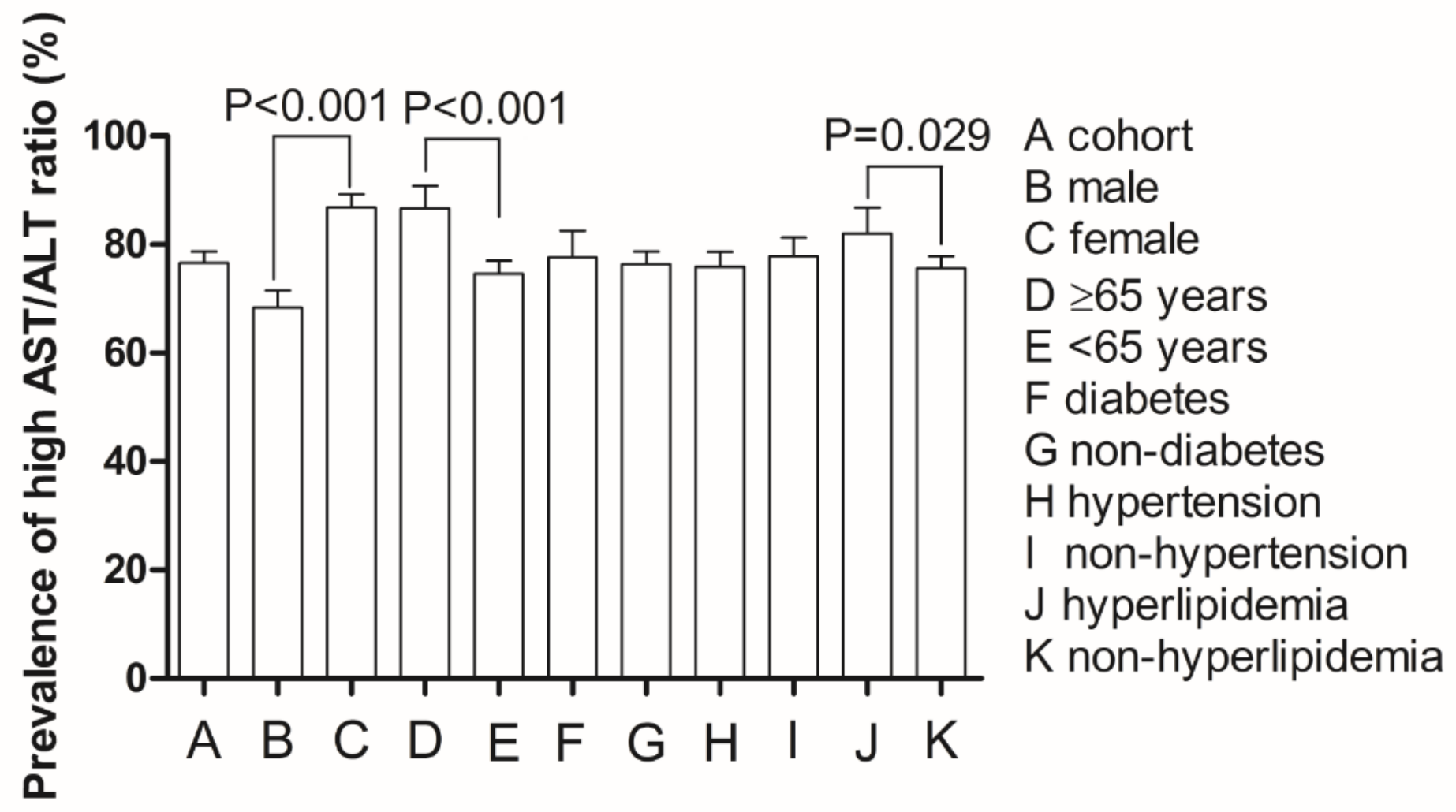

Figure 2

Prevalence of the high AST/ALT ratio in the cohort population and subgroups AST/ALT, aspartate aminotransferase/alanine aminotransferase. 


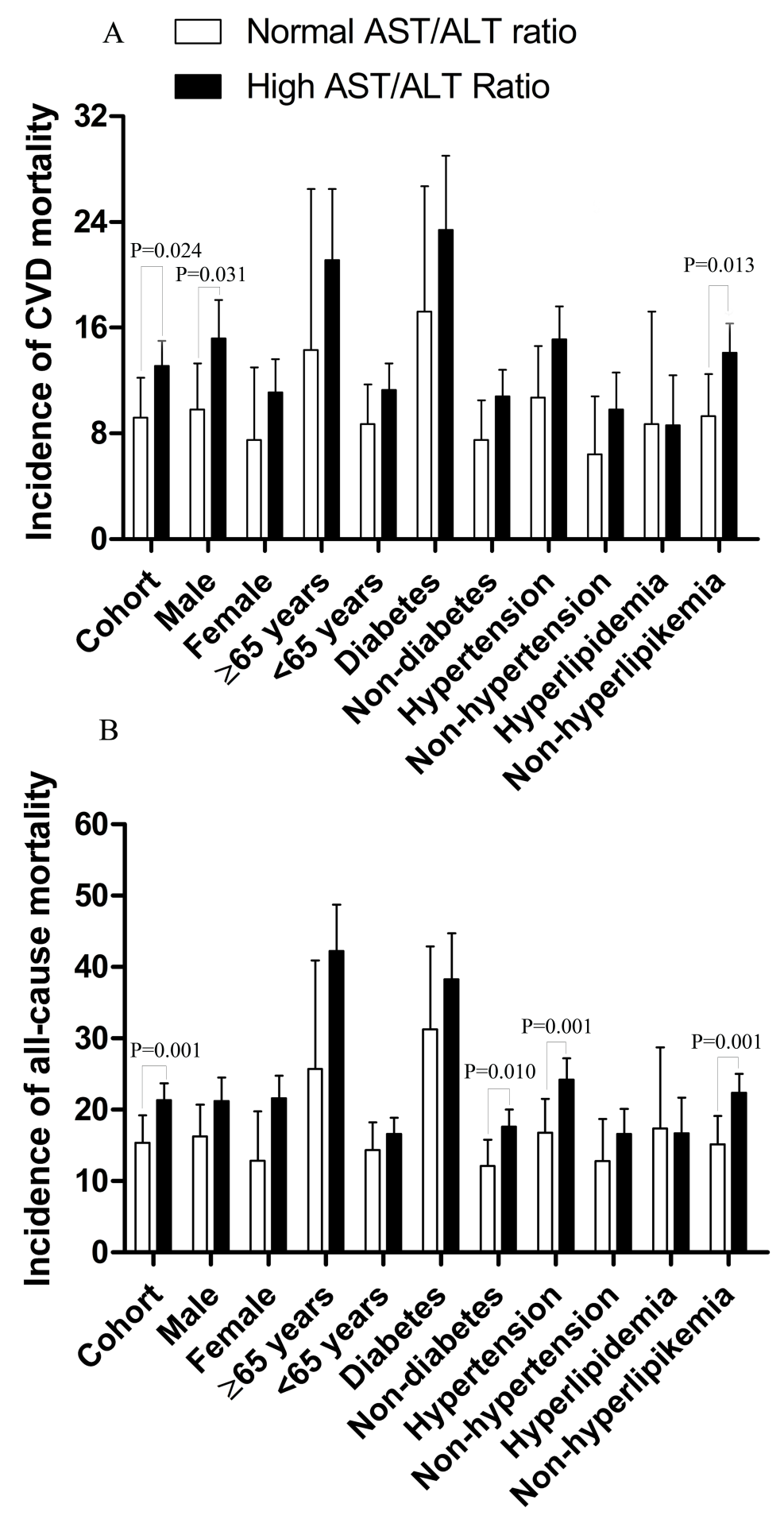

Figure 3

CVD and all-cause mortality incidence in the cohort population and subgroups A, CVD mortality incidence rates; $\mathrm{B}$, all-cause incidence rates. AST/ALT, aspartate aminotransferase/alanine aminotransferase; CVD, cardiovascular disease. 

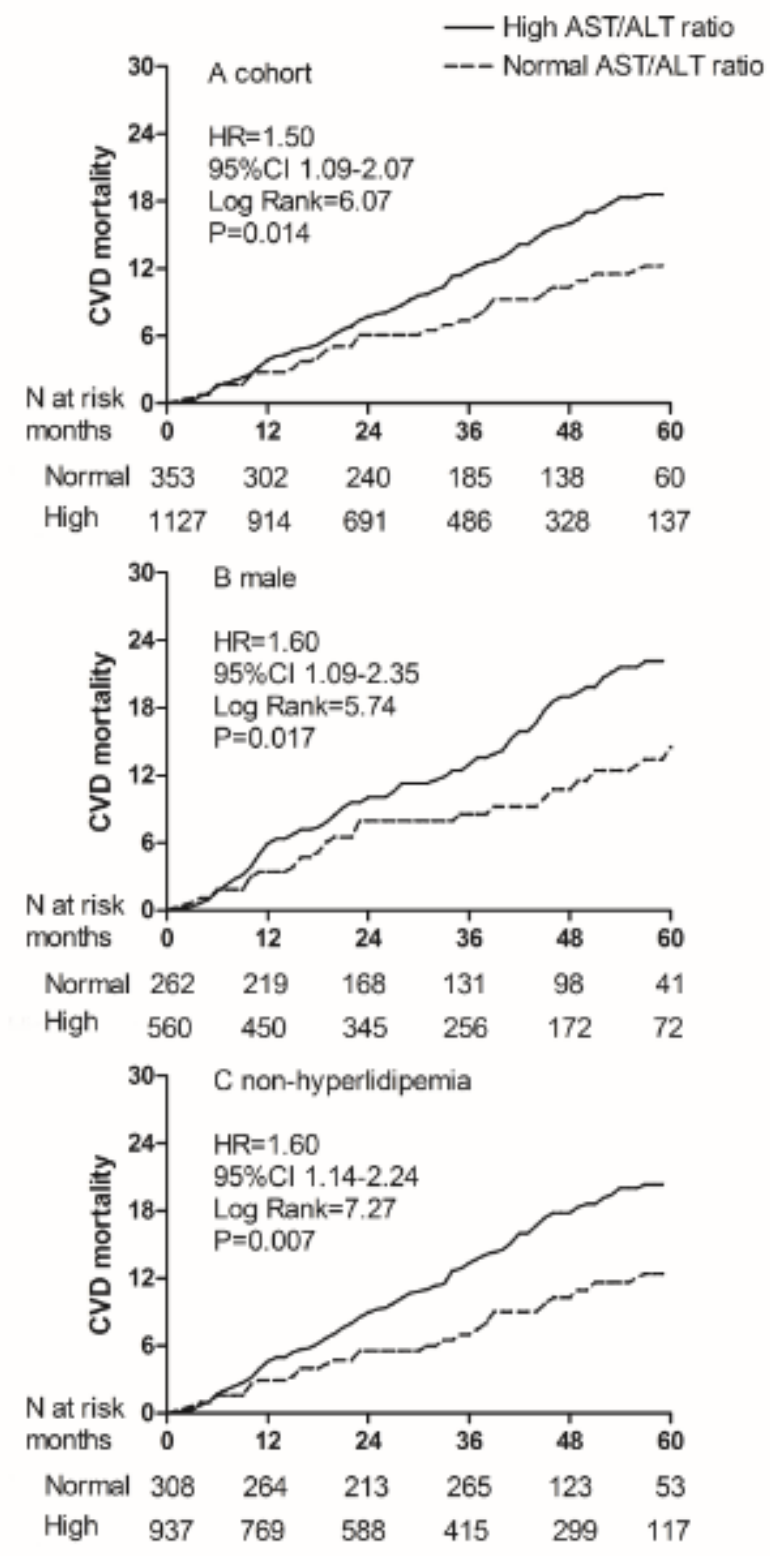

\section{Figure 4}

Cumulative CVD mortality curves in the cohort population and subgroups A, cohort; B, male; C, nonhyperlipidemia. AST/ALT, aspartate aminotransferase/alanine aminotransferase. CVD, cardiovascular disease. 

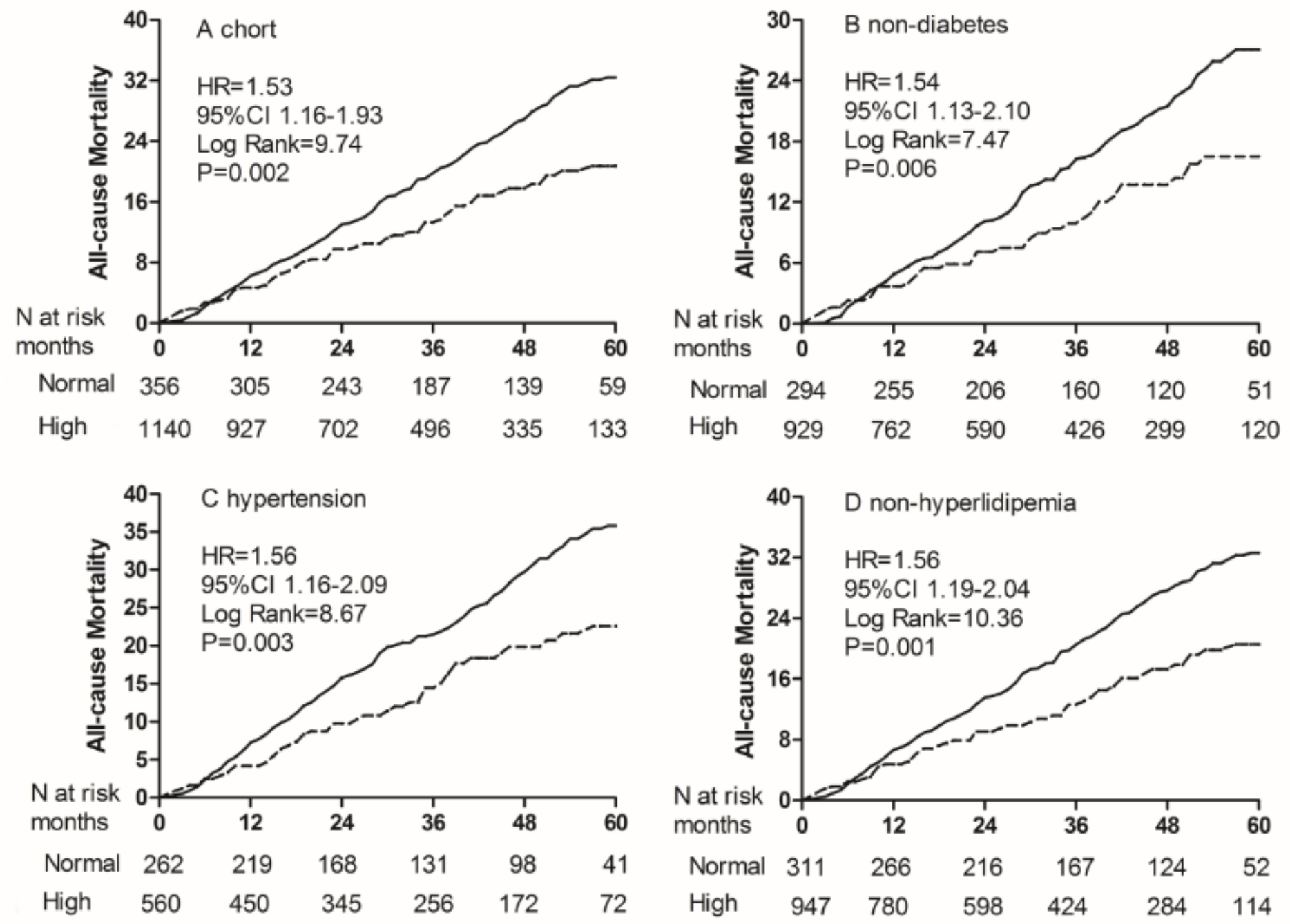

\section{Figure 5}

Cumulative all-cause mortality curves in the cohort population and subgroups A, cohort; B, non-diabetes; C, hypertension; D, non-hyperlipidemia. AST/ALT, aspartate aminotransferase/alanine aminotransferase. 

A Cox regression model
B Cox regression model
CVD mortality
All-cause mortality

High AST/ALT ratio vs.normal AST/ALT ratio (as a reference) High AST/ALT ratio vs.normal AST/ALT ratio (as a reference)
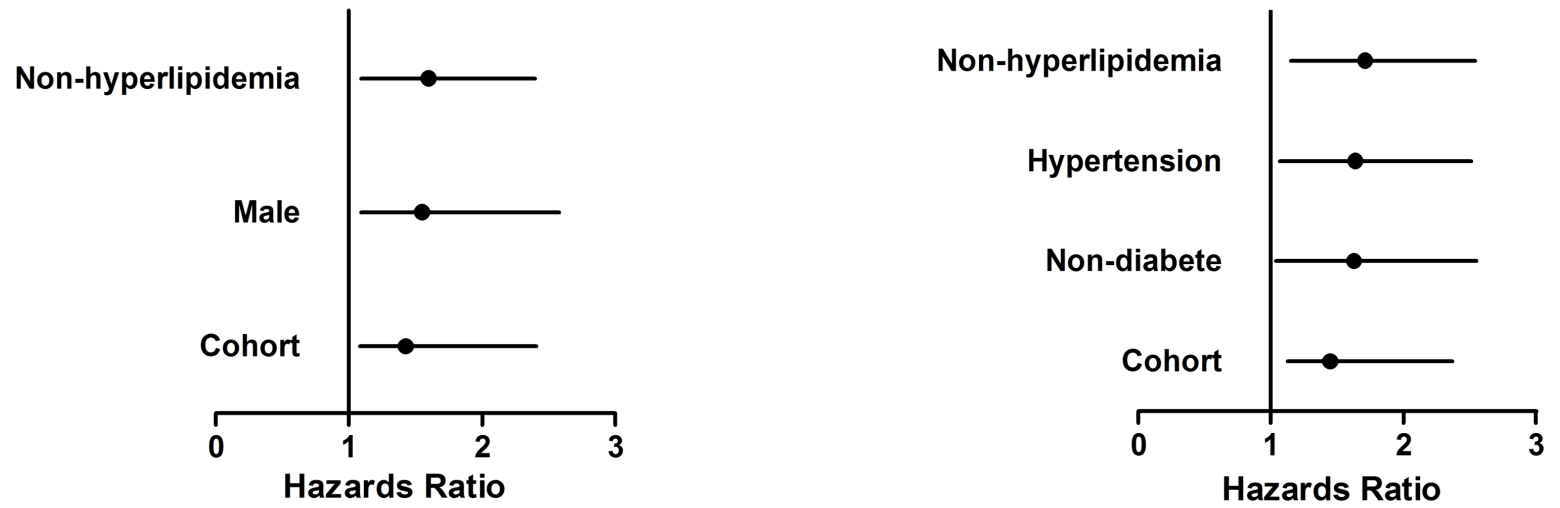

Figure 6

Adjusted HR for CVD and all-cause mortality with the Cox regression models. AST/ALT, aspartate aminotransferase/alanine aminotransferase. CVD, cardiovascular disease. 